\title{
LA CATEGORIA DE ESPACIO EN LA FÍSICA ATÓMICA
}

\section{Presentación del problema}

Los categorías de una ciencia son los conceptos fundamentales implicados necesariamente en todo juicio establecido dentro de dicha disciplina. Por ello, las categorías sirven como discriminantes para acotar el dominio a que corresponde un conocimiento, puesto que expresan las formas comunes de existencia de los procesos estudiados por la disciplina en cuestión. Por su carácter general y por las múltiples conexiones que enlazan a las formas de existencia expresadas en las categorías, éstas constituyen los elementos del sistema que sirve de estructura al conocimiento científico. Este sistema de las categorías es flexible y eminentemente dinámico, porque sus elementos se transforman dialécticamente sin cesar. El avance del conocimiento hace que se multipliquen ininterrumpidamente las categorías, para caracterizar definidamente las nuevas manifestaciones de lo existente. A la vez, con estas determinaciones se enriquece el conocimiento de las relaciones de unos procesos con otros, dando por resultado una interconexión cada vez mayor entre las categorías que expresan sus propiedades más generales y el consiguiente ensanche del sistema en amplitud y en profundidad. Por otro lado, el desarrollo de la ciencia plantea constantemente la necesidad de modificar las categorías ya establecidas $y$, llegado el caso, conduce a la substitución de unas categorías por otras, cuando se pone al descubierto que las primeras solamente representan aspectos limitados e insuficientes de propiedades objetivas de mayor generalidad. En ciertos períodos, cuando se exploran científicamente nuevos niveles de la realidad, el proceso de modificación de las categorías opera con mayor rapidez. Yero, aunque de modo más lento, en todos los períodos tenemos que el progreso del conocimiento va poniendo de manifiesto la necesidad de modificar las categorías, hasta que la acumulación de estos cambios graduales es suficiente para imponer, de manera indispensable, una transformación radical en ciertas categorías. Esta transformación ocurre siempre en forma espectacular y provocando una crisis que conmueve a la ciencia de que se trate en su totalidad.

De esta manera, advertimos como peculiaridad distintiva de las categorías su flexibilidad para el cambio y la transformación. Porque como cada categoría refleja un determinado aspecto de la existencia y su sistema representa al conjunto de la existencia en un cierto nivel, entonces, las categorías se enlazan entre sí, se condicionan recíprocamente, se oponen, se interpenetran, 
se truecan unas en otras y se transforman sin cesar, para reflejar a la existencia en su movimiento y en su integridad. Por lo tanto, toda formulación que de ellas se haga será transitoria, aunque se mantenga dentro de una invariancia relativa; ya que, tarde o temprano, el avance de la ciencia impondrá su modificación. Y esta conversión llega a ser de tal manera profunda que, en ocasiones, lo único que se conserva de las anteriores categorías es el nombre.

Ahora bien, al mismo tiempo que tenemos categorías peculiares de cada ciencia, también existen otras categorías que abarcan grupos de ciencias y otras más que se refieren al conocimiento científico entero; porque representan propiedades de un sector más amplio de la realidad o de todos los procesos existentes. Debido a esto, en cada ciencia son implicadas a la vez sus categorías exclusivas y las categorías más generales del conocimiento científico. Sin embargo, esta aplicación universal de las categorías del conocimiento en su conjunto no significa que en cada uno de los dominios particulares tengan exactamente el mismo carácter. En realidad, las categorías generales adoptan ciertas formas peculiares cuando son consideradas dentro de un campo particularizado. Así, por ejemplo, la categoría de espacio es diferente, en cierto modo, dentro del dominio de las ciencias sociales que en el de las ciencias naturales $y$, dentro de este campo, tiene distintas especificaciones en la geometría, en la física, en la química y en la biología. Y, aun dentro de los límites de una disciplina determinada, como la física, la categoría de espacio presenta variaciones notables en cada una de sus ramas componentes. En consecuencia, cada una de las divisiones de la ciencia posee sus categorías distintivas y las categorías generales especificadas peculiarmente dentro de su dominio, y ellas la definen conjuntamente por completo. Pero, al mismo tiempo, las categorías generales se determinan en el desarrollo de las investigaciones específicas de cada ciencia y de todas sus ramas y, por lo tanto, su determinación general está condicionada por las caracterizaciones particulares que adopta en los distintos campos científicos. Tomando en cuenta lo anterior, intentamos presentar la caracterización adquirida por uno de los conceptos fundamentales del conocimiento, la categoría de espacio, dentro del ámbito tan fecundo y activo de la física atómica.

\section{Situación actual de la teoría cuántica}

La necesidad de pasar de la física clásica a la mecánica cuántica, para explicar los procesos atómicos, fue tan imperiosa y objetiva como la que obligó a superar la propia física clásica con la teoría de la relatividad, para explicar los procesos astronómicos. En ambos extremos de la experiencia, el punto de partida lo ofreció el descubrimiento de la existencia de valores críticos, antes insospechados, para ciertas propiedades físicas. En la teoría de la relatividad, la propiedad destacada de este modo es la velocidad y su valor crítico es $c$, la 
velocidad de la luz. En la mecánica cuántica, la propiedad singular es el cambio en la acción ${ }^{1}$ y su valor crítico es $h$, el cuanto descubierto por Planck. Así, la relatividad reduce la velocidad de lo infinitamente grande a un máximo sumamente grande ${ }^{2}$ pero finito. Y, por su parte, la mecánica cuántica restringe los cambios en la acción de lo infinitamente pequeño a un mínimo extremadamente pequeño, ${ }^{3}$ pero finito también. Pues bien, desarrollada sobre la base de la discontinuidad manifiesta en el cuanto - dosis mínima indivisa-, la teoría cuántica ha permitido generalizar las explicaciones de una gran cantidad de resultados experimentales, haciendo comprensibles numerosos procesos físicos que no lo son conforme a las leyes clásicas y abriendo la posibilidad de anticipar racionalmente el descubrimiento de procesos nuevos. Además, ha formulado muchas leyes importantes acerca del comportamiento de las partículas elementales y constituye el fundamento científico de gran número de realizaciones técnicas sorprendentes y enormemente promisorias en sus usos pacíficos.

Sin embargo, después de un período de intensa investigación sobre los procesos atómicos y sus correspondientes conceptos, junto con el descubrimiento de las nuevas leyes físicas que rigen su comportamiento, la mecánica cuántica ha pasado por una etapa exclusivamente experimental, en la cual no se hizo nada esencialmente nuevo respecto al examen de los principios que le sirven de fundamento. Al contrario, dichos principios fueron considerados como dogmas absolutos e intocables de una especie de "ortodoxia cuántica". Por fortuna, en la física atómica existe actualmente una corriente, cada vez más poderosa, que persigue el propósito de superar las inconsecuencias y oscuridades de esa "ortodoxia". En este empeño confluyen ahora los trabajos de David Bohm, Janossy, Blojinzev, Frenkel, De Broglie, Terletski, Vigier, Takabayasi y otros, coincidiendo con los esfuerzos anteriores de Einstein, Planck, Lorentz, Langevin y Schroedinger, quienes nunca aceptaron la mecánica cuántica como una teoría física completa. ${ }^{4}$ Todavía no se puede decir que se hayan resuelto íntegramente las dificultades que presenta el conseguir la consecuencia racional y la comprobación experimental para alguna de las

1 La acción se define físicamente como el producto dimensional de la energía por el tiempo.

2 Velocidad de la luz: $\mathrm{c}=2.99776 \times 10^{10} \mathrm{~cm} / \mathrm{seg}$.

3 Constante de Planck: $h=6.6237 \times 10^{-27} \mathrm{erg} \cdot \mathrm{seg}$.

4 Para obtener información sobre estos trabajos, pueden consultarse las siguientes exposiciones: Louis de Broglie et Jean P. Vigier, La physique quantique restera-t-elle indéterministe?, Gauthier-Villars, Paris, 1953; Questions scientifiques, physique, Les Editions de la Nouvelle Critique, Paris, 1952; Albert Einstein, The meaning of relativity, Princeton University Press, Princeton, 1953; Louis de Broglie, El problema de la interpretación causal y abjetiva de la física cuántica, Universidad Nacional de México, Suplemento del Seminario de Problemas Científicos y Filosóficos, México, N⿳4 4, 1956; L. Janossy, "The physical aspects of the wave-particle problem", Acta Physica Hungarica, 1, 1952; págs. 423-467; Albert Einstein: philosopher-scientist, Ed. P. A. Schilpp, The Library of Living Philosophers, Evanston, Ill., 1949; E. Schroedinger, "Are there quantum jumps?", The British Journal for the Philosophy of Science, Vol. III, 1952; págs. 109-123, 233-242. 
nuevas teorías propuestas. Es claro que no se puede desechar la posibilidad de que estas tentativas terminen en el fracaso; pero, también es indudable que con estas reinterpretaciones se tiene la posibilidad de llegar a resultados nuevos y fructuosos. En todo caso, lo que sí ya es imposible es el seguir sosteniendo la teoría cuántica "ortodoxa".

La teoría cuántica actual tiene tres presentaciones -el método de las matrices de Heisenberg, la mecánica ondulatoria de De Broglie y Schroedinger y la mecánica cuántica relativista de Dirac- que, a pesar de sus apaientes divergencias, no se encuentran últimamente en conflicto. Por el contrario, son equivalentes matemáticamente y solo difieren en la disposición conceptual de que están revestidas y en sus ventajas o desventajas para abordar algunos problemas. Además, los tres métodos conducen a los mismos resultados acertados y llevan a absurdos semejantes. Las tres interpretaciones constituyen un refinamiento de la mecánica cuántica primitiva $\mathrm{y}$, como se pueden utilizar indistintamente, forman lo que se llama la teoría cuántica "ortodoxa". Sus nociones primarias están construídas por concepciones híbridas entre las "partículas materiales" de Newton -adecuadamente ajustadas o desconcertadas por los postulados cuánticos- y un aparato matemático complicado determinado principalmente por consideraciones estadísticas - de un tipo diferente a las que constituyen la estadística clásica-. Pero, con todo, la teoría cuántica contiene serias dificultades internas, que indican seguramente faltas de consecuencia y de integridad en sus "principios" y en sus desarrollos; lo cual significa que la estructura lógica de la teoría es imperfecta. Se puede decir, incluso, que el carácter "ortodoxo" de la teoría cuántica se ha hecho tan complejo y poco satisfactorio como el que exhibía la mecánica clásica cuando se trataba de aplicarla inflexiblemente a la explicación de los procesos atómicos.

Lo que sí se encuentra completamente esclarecido por los descubrimientos experimentales es el hecho de que la mecánica clásica es inaplicable al dominio de los procesos atómicos. Sin embargo, la teoría cuántica simplemente plantea el problema de hallar explicación al comportamiento de las partículas elementales y prepara el terreno para arribar al establecimiento de nuevas concepciones físicas; sin que, por ello, la propia teoría cuántica haya resuelto ya estas cuestiones. Entonces, es imprescindible hacer la revisión rigurosa de los problemas planteados, atacándolos en dos frentes: primero, acumulando el material experimental de los hechos que no se pueden explicar satisfactoriamente; segundo, criticando las bases de los argumentos que han llevado a establecer la "ortodoxia" cuántica, que ahora se muestra tan insuficiente como la física "tradicional". El primer frente pertenece propiamente a los físicos experimentales, mientras que en el segundo es necesaria la colaboración y el esfuerzo común de los físicos teóricos y de los filósofos; pero en ambos frentes se requiere la conjugación de la teoría y de 
la práctica científicas. Desde luego, se puede afirmar que la nueva interpretación debe ser coherente internamente, debe incluir e iluminar el conocimiento experimental que ya se tiene de las partículas elementales, debe resolver todas las paradojas advertidas, debe hacer que el nivel atómico de la realidad sea congruentemente inteligible y manejable y, sobre todo, debe abarcar la explicación del desarrollo, del origen, de las complejas interacciones y de las transformaciones de las partículas elementales. ${ }^{5}$

\section{Necesidad de establecer nuevas categorías}

Como lo decíamos antes, Einstein, Planck y Langevin tuvieron siempre a la mecánica cuántica por una teoría incompleta y consideraron que la introducción de los nuevos conceptos indispensables para integrarla permitiría demostrar, entre otras cosas, el determinismo estricto entre las partículas elementales. Esta consideración implica que nuestra actual impotencia para explicar los procesos atómicos se deba al empleo extralógico de los conceptos clásicos - construídos a partir de nuestros conocimientos experimentales de los procesos macroscópicos- que han sido trasladados sin crítica ni modificación algunas al nivel microscópico, como elementos para su descripción. Por ende, una parte de la solución parece encontrarse en la transformación de las categorías de espacio, tiempo, masa y energía, interpretando con fruto y rigor los hechos conocidos experimentalmente sobre las partículas atómicas. En realidad, las previsiones que se pueden derivar de una teoría están restringidas por las categorías que la propia teoría acepta como válidas. Entonces, cuando las categorías de una teoría son deficientes, la teoría puede ser incapaz de prever ciertos acontecimientos con precisión, aun cuando dichos acontecimientos sean enteramente reales y aunque ocurran de un modo perfectamente determinado. Cuando sucede esto, se considera a la teoría en cuestión como incompleta. ${ }^{6}$ Este es el caso de las teorías cuánticas "ortodoxas", puesto que limitan su campo a los procesos colectivos formados por conjuntos enormes de partículas elementales, sin que puedan ofrecer explicaciones satisfactorias acerca de los procesos en que intervienen partículas elementales aisladas o grupos pequeños de ellas. Por lo tanto, es posible que la vaguedad de la mecánica cuántica - puesta en evidencia por las relaciones de incertidum-

5 Las partículas elementales conocidas hasta ahora son: el fotón; los leptones, que incluyen al neutrino, al electrón negativo y al electrón positivo; los mesones, en sus varios tipos $\mu, \pi, \theta, \tau$ y $\mathrm{K} ;$ y los bariones, que incluyen al protón positivo, al protón negativo, al neutrón y a los hiperones $\Lambda, \Sigma$ y de cascada.

6 Así, por ejemplo, la termodinámica es una teoría incompleta, porque limita su dominio a las categorías y las magnitudes macroscópicas, sin poder suministrar información sobre los procesos microscópicos. En cambio, en la teoría cinética los conceptos macrosć́picos de la termodinámica - tales como la temperatura, la presión y la entropía- son substituídos por los conceptos mecánicos aplicables a los procesos individuales, para poder dar cuenta y razón de ellos. 
bre- se deba simplemente a que la teoría es incompleta. Con todo, es necesario advertir que, en rigor, es imposible construir teoría alguna que fuese enteramente completa; ya que eso representaría que su dominio abarcara por entero a la realidad, sin tener límite alguno. ${ }^{7}$ Por consiguiente, lo que se debe plantear es la formulación de una teoría cuántica que sea lo suficientemente completa como para ofrecer una explicación racional y objetiva de todos los conocimientos logrados sobre los procesos atómicos. $\mathrm{Y}$, cuando se consiga estructurar tal teoría se tendrá a la vez -como lo demuestra reiteradamente la historia de la ciencia - una explicación estricta para una enorme multitud de hechos que se descubrirán en el futuro; ya que toda generalización científica bien fundada supera la extensión y la intensidad de sus cimientos.

Según parece, ni los conceptos mecánicos clásicos ni las categorías de campo pueden servir, tal como están formulados, para superar la teoría cuántica actual. Es necesario establecer categorías radicalmente nuevas, que diferirán de las conocidas hasta ahora. Además, las nociones empleadas "cuánticamente" se han hecho demasiado flúidas en su aplicación. Por ello, es menester encontrar una definición suficientemente neta de los conceptos que se emplean; sin olvidar que se tratará, por de pronto, de nociones extrañas al llamado "sentido común", que tendrán un carácter hipotético mientras no puedan ser verificadas satisfactoriamente en los experimentos. Por otra parte, es preciso tener en cuenta que el significado objetivo de la física cuántica, a pesar de todos sus éxitos sorprendentes, no ha sido esclarecido tan profundamente como sí se ha hecho, en cambio, con las nuevas categorías implícitas en la teoría de la relatividad. En consecuencia, es indispensable examinar con claridad la mecánica cuántica y poner al descubierto todas las nociones contenidas en ella, para someterla luego a una crítica rigurosa y poder planear así la construcción lógica de las nuevas categorías físicas. Para conseguir esto se debe practicar un análisis filosófico penetrante de la teoría cuántica actual. Y, por lo demás, ante las pruebas tan convincentes aportadas por los resultados experimentales, es necesario aprender a servirse de las nuevas posibilidades dialécticas de pensar que la misma naturaleza nos procura, tal como ha sido puesto de manifiesto en los conocimientos adquiridos acerca de las partículas atómicas.

Está enteramente en claro que la formulación de las nuevas categorías cuánticas no puede consistir en la simple yuxtaposición de dos o más categorías clásicas, para considerarlas en su simple reunión. Tal manera de proceder

7 Aun suponiendo que hubiera la posibilidad de formular una teoría completa, de acuerdo con todos los conocimientos adquiridos hasta un momento dado, no obstante, dicha teoría se mostraría más adelante como incompleta, cuando se descubrieran otros aspectos de la realidad que excedieran el dominio acotado por la teoría en cuestión y que ni siquiera se sospecharan con arreglo a dicha teoría. 
ha conducido a dificultades tremendas; como las que resultan, por ejemplo, del empeño de sostener a la vez, sin conjugación íntima y manteniendo su separación absoluta, los conceptos clásicos de onda y de corpúsculo como aspectos mutuamente excluyentes de las partículas elementales. ${ }^{8}$ También se encuentra obviamente aclarado que las contradicciones mostradas en estas reuniones formales de conceptos clásicos son completamente superables, siempre que su solución se busque por el camino de establecer su síntesis dialéctica con base en la interpenetración y en la unidad de los opuestos, interpretando de manera racional y objetiva los resultados experimentales. Por lo tanto, hay que transformar dialécticamente ciertas categorías clásicas, abandonando otras y estableciendo también otras nuevas, hasta poder formular una teoría cuántica que corresponda efectivamente a la realidad y que sirva como instrumento para planear muchos descubrimientos nuevos.

El pasado inmediato de la ciencia física nos enseña algunos de los posibles caminos a seguir. Uno de ellos es el de la teoría de la relatividad. Anteriormente, el campo eléctrico y el campo magnético eran considerados por separado y como distintos. La relatividad fundió ambos aspectos para introducir el continuo espacio-tiempo tetradimensional, estableciendo el tensor electromagnético de cuatro dimensiones que está situado en este nuevo marco. Tal vez será necesario hacer algo análogo para resolver dialécticamente el antagonismo de las dualidades onda-corpúsculo, continuidad-discontinuidad, espacio-cantidad de movimiento, energía-tiempo, espacio-tiempo, espacio-energía, tiempo-cantidad de movimiento y energía-cantidad de movimiento, que se plantean agudamente en la mecánica cuántica. Otra posibilidad consiste en intensificar la exploración de las posibilidades que ofrece la formulación rigurosa de la teoría cuántica del campo; porque, en ella, las partículas elementales aparecen como cuantos de su correspondiente campo -o sea, como singularidades discontinuas de un medio continuo, al cual se encuentran ligadas indisolublemente-, sin que sea necesario introducir en la teoría, a título complementario, las hipótesis del "corpúsculo" clásico o de la "onda" clásica.

Una posibilidad más la tenemos en el procedimiento seguido para formular las cuatro interpretaciones del dualismo onda-corpúsculo: 1) Considerar la existencia de las ondas, en forma análoga a su concepción clásica, teniendo a las manifestaciones corpusculares como meras apariencias, como lo sostiene la hipótesis de Schroedinger. 2) Considerar al corpúsculo como real y a la onda como mera abstracción que guía su movimiento, que es la hipótesis de la onda piloto de De Broglie. 3) Considerar a la onda y al corpúsculo como simples apariencias complementarias sin realidad física

8 Tanto la representación literalmente corpuscular, a la manera clásica, como la representación latamente ondulatoria, también al modo clásico, de las partículas atómicas, acusan claramente su carencia de validez a través de las relaciones de incertidumbre. 
alguna, como se desprende en último extremo de la hipótesis de Bohr. 4) Considerar que tanto la onda como el corpúsculo tienen realidad, siendo el corpúsculo una singularidad en el seno de un fenómeno ondulatorio amplio, como hace De Broglie en su hipótesis de la doble solución. Y, por lo tanto, después de considerar todos los tipos posibles de solución, empeñarse en desenvolver la que resulte estar más de acuerdo con las exigencias científicas de encontrar una explicación racional y objetiva de la realidad. En el caso ilustrado, tendríamos el proyecto de llegar a fundamentar el establecimiento de un juicio de reciprocidad, ${ }^{9}$ en el cual se sintetizaran dialécticamente la noción de onda y la noción de corpúsculo. Tal juicio se podría enunciar elementalmente así: Todo corpúsculo se mueve ondulatoriamente y todo movimiento ondulatorio corresponde a un corpúsculo. Por supuesto, para culminar este procedimiento habría que estudiar luego todas las posibilidades de generalización y de análisis objetivo de cada dualidad contradictoria.

Por otro lado, tenemos que las categorías deben quedar expresadas en forma relativamente flexible, para ser aplicables fructuosamente a la realidad y para que ofrezcan constantemente susceptibilidad a las modificaciones que se hagan necesarias. ${ }^{10}$ Hay que tener presente que la realidad acaba siempre por desbordar los cuadros construídos por nuestra razón. Por ello, de modo permanente, tenemos que atender a las divergencias que se suscitan entre la realidad siempre infinitamente compleja, indefinidamente matizada y continuamente en transformación, por una parte, y nuestra razón que construye conceptos más o menos rígidos, más o menos esquemáticos y siempre incompletos, por otra parte. Ahora bien, las categorías son susceptibles de aplicarse a la realidad en la medida en que la reflejan de manera definida y en cuanto se verifica su correspondencia con ella. Pero nunca podemos esperar que tal correspondencia se mantenga invariablemente hasta el final. El espíritu lógico y sistemático es indispensable, porque nos permite precisar nuestras ideas y nuestros razonamientos $y$, sin él, nuestros conocimientos permanecerían siempre como nociones vagas y meramente cualitativas. Pero también es ineludible el espíritu crítico y objetivamente dialéctico, para poder interpretar con acierto y corregir las construcciones lógicas; ya que la realidad es demasiado flúida e inagotablemente rica, como para que jamás se pueda encerrar por entero en el marco rígido y abstracto de nuestras representaciones categoriales y de nuestros sistemas funcionales.

9 Este tipo de juicio constituye un dilema, cuyas alternativas serían, para el caso de las partículas elementales: a. ser corpusculares y ondulatorias a la vez; y, b. no ser corpusculares ni ondulatorias y, por lo tanto, no ser partículas elementales (Cf. Eli de Gortari, Introducción a la lógica dialéctica, Fondo de Cultura Económica, México, 1956; págs. 151-155, 166).

10 Cuando un concepto cualquiera se precisa hasta el extremo y se postula como absoluto e inmutable, se convierte irremediablemente en una mera forma idealista sin contenido real y sin extensión alguna; como ocurre, por ejemplo, con el concepto metafísico de "ser". 


\section{Requisito de correspondencia}

Tal como la teoría de la relatividad se convierte en la física clásica cuando las velocidades son pequeñas en comparación con la velocidad de la luz, así también, la mecánica cuántica se transforma en la física clásica cuando los cambios cuánticos en la acción son pequeños en comparación con la acción total presente. ${ }^{11}$ Este hecho -conocido con el nombre de principio de correspondencia - nos muestra con claridad cómo las teorías científicas comprobadas ampliamente en los experimentos no sufren detrimento alguno en su validez cuando se descubren procesos nuevos que ya no pueden ser abarcados por ellos. En tal caso, se descubren sencillamente las fronteras de dichas teorías y se determina con precisión su dominio limitado, dentro del cual siguen siendo plenamente válidas. A la vez, esta correspondencia entre las teorías físicas plantea la exigencia de que en los casos limitantes, de los procesos atómicos considerados en grande - es decir, en enormes conjuntos cuyas dimensiones totales son comparables a las humanas-, las propiedades cuánticas se conviertan completamente en propiedades clásicas. Y, lo que es más, del mismo modo como se puede observar una transición simultáneamente gradual y brusca entre las leyes clásicas y las leyes relativistas, igualmente existe una transición continua y discreta a la vez entre las leyes de la física clásica y las leyes de las partículas elementales. Más aún, incluso existen ciertas leyes clásicas que son invariantes respecto a la transformación de la mecánica newtoniana en mecánica cuántica; o sea, que algunas leyes clásicas son enteramente válidas -sin modificación alguna- para explicar el comportamiento de las partículas atómicas. Entre estas leyes invariantes tenemos, por ejemplo, al principio de la conservación de la energía y de la cantidad de movimiento. ${ }^{12}$

Pues bien, en nuestro análisis de la categoría de espacio en la física atómica, debemos tener en cuenta la posibilidad de que las características del espacio en el dominio de las dimensiones medianas, que es estudiado por la física clásica, resulten expresiones aproximadas de las características de las propiedades espaciales de las partículas elementales. Asimismo, es posible que algunas cualidades del espacio en términos astronómicos también se muestren como aproximaciones a las cualidades espaciales de los leptones,

11 Estas conversiones representan dos casos conspicuos de cumplimiento de la ley dialéctica de la transformación de la cantidad en cualidad. El cambio gradual en la magnitud de las dimensiones de los procesos, desde el nivel ultramicroscópico hasta el de las dimensiones comparables a las humanas, produce la transformación brusca de las leyes cuánticas a las leyes clásicas. Y, a su vez, el acrecentamiento continuo de la magnitud de las dimensiones hasta alcanzar el nivel astronómico, trae aparejada la conversión súbita de las leyes clásicas en las leyes relativistas. Y lo mismo sucede cuando el cambio cuantitativo de las dimensiones se realiza en sentido opuesto.

12 Esta ley también es invariante respecto a la transformación de la mecánica clásica en mecánica relativista. 
mesones y bariones. $\mathrm{Y}$, lo que es más, no debe extrañarnos que, en algunos casos, nuestro examen nos lleve a concluir la invariancia de ciertos rasgos del espacio macroscópico en el nivel microscópico. Por supuesto, las propiedades espaciales verificadas objetivamente en el dominio macroscópico nos servirán fructuosamente como hipótesis para explorar el espacio en las dimensiones cuánticas, hasta el momento en que surja la necesidad de modificarlas o de desecharlas, por imposición de los hechos. Es decir, que al tratar de construir la categoría de espacio para los fenómenos atómicos, indagaremos la intexpretación de lo desconocido en términos de lo conocido, pero, sin aferrarnos incondicionalmente a lo conocido. Por otro lado, tendremos que conseguir finalmente que la categoría cuántica de espacio se transforme estrictamente en la categoría clásica de espacio, cuando los procesos atómicos se presenten en gran escala, o sea, en la escala de las dimensiones humanas. En estas condiciones quedará cumplido el requisito científico de la correspondencia entre las categorías.

\section{Posición espacial y cantidad de movimiento}

Como es sabido, uno de los descubrimientos más sorprendentes hechos por la teoría de la relatividad restringida es el esclarecimiento de que la simultaneidad de dos acontecimientos ocurridos en diferentes lugares es enteramente problemática. Análogamente, en el caso de los procesos atómicos, se ha puesto al descubierto cómo se vuelve impreciso el significado de la determinación simultánea de la posición definida y la cantidad de movimiento también definida de una partícula elemental. Tal como lo ha establecido con exactitud Heisenberg en la expresión de sus relaciones de incertidumbre, mientras más exactamente se determina la posición de una partícula, más incierta resulta ser su cantidad de movimiento; y, recíprocamente, mientras más precisamente se determina la cantidad de movimiento de una partícula, más incierta resulta ser su posición. ${ }^{13}$ Todavía con más exactitud, mientras más precisamente se determina la posición de una partícula elemental respecto a una de las dimensiones espaciales, más incierta resulta ser la determinación de la componente de la cantidad de movimiento en esa misma dimensión -esto es, de la representación de la cantidad de movimiento en la misma dirección del espacio-. No obstante, es necesario advertir que es completamente posible determinar la posición de una partícula con el grado de precisión que se quiera; y que, igualmente, es enteramente posible de-

1.3 Entre las incertidumbres $\Delta x, \Delta y, \Delta z$, de las coordenadas $y$ las incertidumbres $\Delta \mathrm{p}_{\mathrm{x}}, \Delta \mathrm{p}_{\mathrm{y}}, \Delta \mathrm{p}_{\mathrm{z}}$, de las respectivas componentes de la cantidad de movimiento en las mismas direcciones, se cumplen las siguientes desigualdades:

$$
\Delta x \Delta p_{x} \geqslant \frac{h}{4 \pi}, \Delta y \Delta p_{y} \geqslant \frac{h}{4 \pi}, \Delta z \Delta p_{z} \geqslant \frac{h}{4 \pi} .
$$


terminar la cantidad de movimiento de la partícula con toda la precisión que se necesite. ${ }^{14}$ Es decir, que la determinación de la posición espacial de una partícula elemental en una dimensión no afecta en nada a la determinación de la propia posición en esa misma dirección, ni tampoco en cualquiera otra dirección espacial. Igualmente, tenemos que la determinación de la cantidad de movimiento de una partícula elemental en una dirección espacial, no influye en la determinación de la cantidad de movimiento en cualquiera otra dirección, sin exceptuar la primera. Lo único que no es posible es determinar simultáneamente la cantidad de movimiento y la posición de la misma partícula con una precisión infinita. ${ }^{15}$ Desde el punto de vista lógico, tenemos que la relación entre los conceptos de "posición precisamente determinada" y de "cantidad de movimiento precisamente determinada" para una misma partícula elemental y en un mismo tiempo, se puede expresar por medio de un juicio de incompatibilidad. ${ }^{16}$ Por lo tanto, existen tres posibilidades: 1) determinar con precisión la posición, en detrimento de la determinación precisa de la cantidad de movimiento; 2) determinar con precisión la cantidad de movimiento, a costa de la determinación precisa de la posición; y, 3) determinar simultáneamente la posición y la cantidad de movimiento con imprecisión. $^{17}$

Ahora bien, el carácter incierto que asume la determinación simultánea de estas dos magnitudes no se produce, en modo alguno, por el procedimiento empleado en la medición, tampoco resulta de los errores cuantitativos cometidos en la observación de la medida, ni depende del nivel alcanzado actualmente por nuestro conocimiento. En rigor, es la expresión de una propiedad objetiva de las partículas elementales - la de no poseer de manera simultánea un valor estrictamente determinado para su posición espacial y para su cantidad de movimiento-; en lo cual difieren notablemente de las partículas macroscópicas,

14 O sea que, de acuerdo con las mismas relaciones de incertidumbre, cuando se determina la posición de una partícula con toda la precisión requerida, esta determinación no perturba a dicha posición $y$, por consiguiente, al medir inmediatamente después de nuevo la posición de la partícula encontramos a ésta exactamente en la misma posición. Lo que sí habrá cambiado con la determinación de la posición es la cantidad de movimiento de la partícula. Y lo mismo sucede respecto a la cantidad de movimiento que no se altera con la determinación exacta de la propia cantidad de movimiento.

15 La precisión tiene el límite finito, determinado con toda exactitud, de no poder tener una magnitud menor que $\frac{h}{4 \pi}$.

16 En este tipo de juicio se tiene un trilema entre los dos términos conceptuales: a. el cumplimiento de un término junto con el incumplimiento del otro; b. el cumplimiento del otro término acompañado del incumplimiento del primero; y, c. el incumplimiento de un término aparejado con el incumplimiento del otro (Cf. De Gortari, Lógica dialéctica, págs. 146-149, 164).

17 Ya que existe ese límite finito para la precisión que se puede conseguir en esta determinación simultánea, cuyo valor es establecido justamente por las relaciones de incertidumbre. 
que sí muestran dicha propiedad. Asimismo, sería erróneo considerar que la experiencia física actual no fuese lo suficientemente precisa como para que se pudieran medir los verdaderos valores simultáneos de la posición y de la cantidad de movimiento. Por lo contrario, se trata de que la experiencia científica actual es suficientemente precisa para demostrar que esta pareja de magnitudes físicas conjugadas no se realiza objetivamente en las partículas elementales; aun cuando sí se manifieste en ellas con nitidez una u otra de las magnitudes de la pareja, pero siempre por separado. Además, tampoco es acertado considerar esta importante propiedad como prueba o indicio de la existencia de âlguna indeterminación definitiva e insuperable respecto a las propiedades de las partículas elementales o a su comportamiento. ${ }^{18}$ En realidad, las relaciones de incertidumbre no tienen nada de indeterminadas, sino que, al revés, expresan matemáticamente una ley determinada con entera exactitud sobre la correlación indisoluble existente entre la cantidad de movimiento y la posición espacial de las partículas elementales. $\mathrm{Y}$ esta correlación representa una conexión física que explica otras propiedades que son peculiares de las partículas elementales.

El hecho de que los conceptos de posición y de cantidad de movimiento no se puedan atribuir simultáneamente a una misma partícula elemental de un modo preciso y distinto, ha recibido una interpretación arbitraria que se resume en la postulación del llamado "principio de complementariedad" de Bohr. ${ }^{19}$ Desde luego, es necesario aclarar que este "principio" no es, en manera alguna, lo mismo que las relaciones de incertidumbre. Enfocándola lógicamente, la noción de "complementariedad" representa la transformación de las relaciones de incertidumbre, expresables por medio de un juicio de incompatibilidad, en otra conexión conceptual diferente, que se puede expresar como un juicio de exclusion. ${ }^{20}$ Así, las relaciones de incertidumbre son convertidas subjetivamente en una exclusión radical y la imprecisión es transformada de manera absurda en inexistencia. Por consiguiente, las tres posibilidades existentes en la realidad son reducidas inconsecuentemente por la "complementariedad" a sólo dos: 1) la determinación precisa de la posición espacial; y, 2) la determinación precisa de la cantidad de movimiento. Luego, los comple-

18 Un análisis detallado de este problema, tomando en cuenta los trabajos más recientes, se encuentra en mi artículo "El indeterminismo físico en crisis", Cuadernos Americanos, Vol. LXXXII, 4, 1955; págs. 96-114,

10 Un examen actual y pormenorizado de las insuficiencias y de las falsas generalizaciones contenidas en este "principio", lo tenemos en el artículo de Mario Bunge, "Strife about complementarity", The British Journal for the Philosophy of Science, Vol. VI, 21 y 26, 1955.

20 En esta clase de juicio se tiene expresada la oposición irreductible entre dos términos conceptuales, en forma de un dilema: si se cumple un término no se cumple el otro, y viceversa; $y$, a la vez, el incumplimiento de un término trae aparejado el cumplimiento del otro, y recíprocamente. De tal manera que queda excluído tanto el cumplimiento simultáneo de ambos términos, como el incumplimiento de los dos términos a la vez. (Cf. De Gortari, Lógica dialéctica, págs. 151-153, 165-166). 
mentaristas hacen una extensión todavía menos fundada de esta supuesta exclusión mutua, considerando que únicamente son posibles en definitiva dos clases de dispositivos experimentales: A. los que permiten determinar la cantidad de movimiento de una partícula elemental; y, B. los que permiten determinar la posición de la misma partícula. La utilización de un dispositivo instrumental de una de estas clases, implica la renuncia completa al empleo de cualquier dispositivo de la otra clase para el mismo caso. Lo que percibimos en un dispositivo experimental -según afirman últimamente los complementaristas - depende de la clase a que pertenezca dicho dispositivo. "Los fenómenos son, en cierto modo, engendrados por las observaciones repetidas." Y, lo que es más, este punto de vista francamente idealista se exagera diciendo que "... no se puede... atribuir a los fenómenos, ni al instrumento, una realidad física autónoma, en el sentido ordinario de la palabra". ${ }^{21}$

Por otro lado, es indispensable aclarar que, no obstante la enorme importancia del hecho expresado por las relaciones de incertidumbre, éstas conciernen únicamente a ciertas parejas de magnitudes físicas. ${ }^{22}$ En cambio, no abarcan propiedades tan fundamentales de las partículas elementales como son la masa, la carga, el $\operatorname{spin}^{23}$ y varias de las manifestaciones de su energía. Esta limitación de las relaciones de incertidumbre hace más patente aún la inconsecuencia de la pretendida generalización establecida por la "complementariedad". En realidad, la interpretación complementarista sostiene a todo trance los conceptos clásicos de posición y de cantidad de movimiento -atribuyéndoles un carácter absoluto e inmutable- para concluir luego que su exclusión recíproca en el caso de las partículas elementales demuestra la incognoscibilidad irremediable de las propiedades de dichas partículas. De manera análoga, insiste en que el único modo de determinar el estado de una partícula es seguir el procedimiento clásico de fijar separadamente y a la vez su cantidad de movimiento y su posición y, luego, considerando la imposibilidad de realizar en las partículas atómicas la medición precisa y simultánea de ambas magnitudes, concluye formalmente el derrumbe del determinismo en este nivel, en la física entera y en todos los dominios de la realidad. ${ }^{24}$ Por otra parte -y esto es lo que más interesa destacar- la noción de "complementariedad" no conduce a ampliación alguna de nuestros conocimientos físicos de la naturaleza, ni tampoco permite formular ninguna

21 Niels Bohr, La théorie atomique et la description des phénomènes, Gauthier-Villars, Paris, 1932; págs. $51,64$.

22 Matemáticamente, estas parejas de magnitudes son aquellas cuyos valores no son conmutativos en el álgebra de las matrices, o sea que el orden en que se toman estos valores como factores sí altera el valor de su producto.

23 Cantidad de movimiento angular de una partícula elemental.

24 En rigor, la mecánica cuántica sí permite determinar con exactitud el estado de un sistema de partículas atómicas, por medio de su "función de estado". Y esta función 'es enteramente determinista. 
hipótesis cuyas consecuencias pudieran ser sometidas a la prueba de la verificación experimental. En suma, el "principio de complementariedad" constituye una generalización extralógica de las relaciones de incertidumbre, que se trata de presentar como su única interpretación posible y que, en lugar de contribuir al análisis que es indispensable hacer para formular objetivamente el significado real de las categorías físicas en el dominio de las partículas elementales, se contenta simplemente con atribuir un carácter absoluto -nada científico- a las concepciones de la mecánica clásica. Y, de este modo, intenta convertir las propiedades fundamentales de la existencia en el nivel atómico, en categorías subjetivas y decididamente irracionales.

Con todo, parece ser conceptualmente imposible determinar la posición y la cantidad de movimiento de una partícula elemental en un mismo instante, con una precisión que exceda a la admitida por las desigualdades que expresan las relaciones de incertidumbre. Sin embargo, si examinamos críticamente las ecuaciones fundamentales de la propia mecánica cuántica, nos encontramos con que la cantidad de movimiento $p$ de una partícula elemental está determinada por la constante cuántica universal $h$, la frecuencia de su movimiento ondulatorio $v-o$ sea, el número de crestas de onda que pasan por un punto en la unidad de tiempo- y la velocidad de la luz $c$, conforme a la relación siguiente: $\mathrm{p}=\frac{\mathrm{h} v}{\mathrm{c}}$. Y, como la frecuencia $v$ es simplemente la relación entre la velocidad de la luz $c$ y la longitud de onda $\lambda$, es decir: $v=\frac{c}{\lambda}$; entonces, despejando el valor de $\lambda$, que es: $\lambda=\frac{c}{v}, y$ substituyéndolo en la ecuación anterior, tenemos: $\mathrm{p}=\frac{\mathrm{h}}{\lambda}$. Desde el punto de vista dimensional, esta ecuación representa: cantidad de movimiento $=$ $=\frac{\text { energía } \times \text { tiempo }}{\text { longitud }}$. Recíprocamente, despejando a $\lambda$ en esta última ecuación, tenemos $\lambda=\frac{\mathrm{h}}{\mathrm{p}}$; que dimensionalmente corresponde a: longitud $=$ $=\frac{\text { energía } \times \text { tiempo }}{\text { cantidad de movimiento }}$. Para el caso de las partículas atómicas que se mueven a velocidades inferiores a la de la luz, el resultado es el mismo. ${ }^{25}$

25 En tal caso, tenemos:

$$
\begin{aligned}
& \text { velocidad ondulatoria de la particula: } \mathrm{V} \\
& \text { cantidad de movimiento: } \mathrm{p}=\frac{\mathrm{hv}}{\mathrm{V}}
\end{aligned}
$$


Además, es necesario tener presente que la validez de la ecuación: $p=\frac{\mathbf{h}}{\lambda}$, se encuentra verificada objetivamente en la realidad con los experimentos de Davisson y Germer, para los electrones, y de Stern, Johnson y otros, para las demás partículas elementales. ${ }^{26}$ Entonces, resulta que el cumplimiento de estas ecuaciones impone la necesidad de tomar en cuenta y operar simultáneamente con la cantidad de movimiento y con una propiedad espacial - como lo es la longitud de onda - de una y la misma partícula elemental. Más aún, las ecuaciones requieren ineludiblemente que se consideren valores precisos de ambas magnitudes; lo cual, de acuerdo con las relaciones de incertidumbre, es justamente imposible hacer con precisión respecto a la cantidad de movimiento y otra propiedad espacial -la posición, fijada también por longitudes- de una y la misma partícula. Nos enfrentamos así a una paradoja insostenible: por un lado, la imposibilidad de hacer la determinación precisa y simultánea de la cantidad de movimiento y de las longitudes que localizan a una partícula espacialmente; por otra parte, tenemos la necesidad, imposible de eludir, de hacer la determinación precisa y simultánea de la cantidad de movimiento y de la longitud de onda -propiedad espacial también- de una partícula elemental. En consecuencia, no sólo es conceptualmente posible, sino que es experimentalmente necesario determinar a la vez, y para una y la misma partícula, la magnitud de su cantidad de movimiento y una de sus magnitudes espaciales. Y, como se advierte claramente, no se trata de que este conocimiento simultáneo se obtenga indirectamente a través de inferencias matemáticas, sino que se obtiene directamente en los experimentos.

Ahora bien, la misma irreductibilidad de la oposición que constatamos plantea abiertamente la urgencia de superar esta paradoja, resolviéndola dialécticamente. Y son los propios términos en que se plantea la contradicción los que nos permiten esbozar su solución. Desde luego, tenemos que considerar la cantidad de movimiento de una partícula elemental como otra forma

$$
\begin{aligned}
& \text { frecuencia: } v=\frac{v}{\lambda} \\
& \text { longitud de onda: } \lambda=\frac{v}{v}
\end{aligned}
$$

de donde se obtiene, por substitución, lo que sigue:

$$
\begin{aligned}
& \text { cantidad de movimiento: } p=\frac{h}{\lambda} \\
& \text { longitud de onda: } \lambda=\frac{h}{p} .
\end{aligned}
$$

26 Los detalles de estos experimentos de difracción de las partículas elementales se pueden encontrar, por ejemplo, en la monografía de J. J. Trillat, Les preuves experimentales de la mécanique ondulatoire, Hermann et Cie., Paris; 1934. 
€n que se manifiesta su longitud de onda, o sea, una propiedad espacial; y, a la vez, tenemos que reconocer en la longitud de onda de una partícula otra manera en la cual se muestra su cantidad de movimiento. En este sentido, el espacio tiene que ser considerado conjuntamente como extensión y como cantidad de movimiento. $O$, más bien dicho, se tiene que reconocer a la cantidad de movimiento y a la extensión como dos formas, diferentes e inseparables, a través de las cuales se manifiesta ese modo de existencia de las partículas elementales que constituye lo que llamamos sus propiedades espaciales. ${ }^{27} \mathrm{El}$ espacio tiene, por lo tanto, dos aspectos que son la extensión y la cantidad de movimiento. Y su relación lógica se puede expresar en función de un juicio de inclusión. ${ }^{28}$ Así, tendríamos el enunciado primordial siguiente: Respecto a las partículas elementales, el espacio se puede manifestar como extensión, o como cantidad de movimiento, o bien, como extensión y cantidad de movimiento a la vez. De esta manera quedarían satisfechas, por una parte, las relaciones de incertidumbre $y$, por otro lado, las ecuaciones que enlazan directamente la cantidad de movimiento con la longitud de onda; lo cual es posible, entre otras cosas, por la coincidencia de la constante cuántica $h$, que es común a los dos grupos de ecuaciones.

\section{Tiempo y energía}

Las relaciones de incertidumbre se aplican también a otra pareja de magnitudes conjugadas: la energía y el tiempo. Es decir, que mientras más precisamente se determina el instante en que una partícula elemental pasa por un punto dado del espacio, más incierta resulta ser la determinación de su energía; y, recíprocamente, mientras más exactamente se determina la energía de una partícula, menos precisa es la determinación del momento en que se encuentra en un cierto punto. ${ }^{29}$ También en este caso es enteramente posible determinar la energía de una partícula con toda la precisión que se quiera; e, igualmente, se puede determinar el instante en que pasa por un punto espacial con cualquier grado de precisión. Lo que no es posible es determinar simultáneamente el tiempo y la energía de la misma

27 Ya la teoría de la relatividad se ha encargado de demostrar antes, dentro de su dominio, que el espacio se encuentra ligado a la cantidad de movimiento por el mismo sistema en que el tiempo está unido a la energía.

$28 \mathrm{El}$ juicio incluyente representa un trilema entre los dos términos conceptuales relacionados, cuyas alternativas son: a. el cumplimiento de un término acompañado del incumplimiento del otro; b. el cumplimiento del otro término junto con el incumplimiento del primero; $y$, c. el cumplimiento simultáneo de ambos términos (Cf. De Gortari, Lógica dialéctica, págs. 146-147, 164).

29 Entre la incertidumbre de la energía, $\Delta E$, y la incertidumbre del tiempo, $\Delta t$, se cumple la siguiente desigualdad: $\Delta E \Delta t \geqslant \frac{h}{4 \pi}$. 
partícula con una precisión infinita. Entonces, se puede expresar asimismo esta relación por medio de un juicio de incompatibilidad. Por consiguiente, existen tres alternativas: 1) determinar con precisión la energía, a costa de la falta de precisión del instante; 2) determinar con precisión el instante, con detrimento de la determinación precisa de la energía; y, 3) determinar simultáneamente la energía y el tiempo con imprecisión. De este modo, entre la energía y el tiempo parece haber la imposibilidad conceptual de determinar el tiempo y la energía de una partícula elemental en un mismo punto del espacio, con una precisión que exceda a la establecida por la desigualdad que expresa la relación de incertidumbre. No obstante, si examinamos críticamente otra de las ecuaciones fundamentales de la mecánica cuántica, nos encontramos con que la energía $E$ de una partícula atómica está determinada justamente por la constante cuántica $h$ y la frecuencia de su movimiento ondulatorio $v-$ que, por representar el número de crestas de onda que pasan por un punto en una unidad de tiempo, es dimensionalmente el inverso del tiempo- conforme a la siguiente relación: $\mathrm{E}=\mathrm{hv}$. Por lo cual resulta que el cumplimiento de esta ecuación impone la necesidad de considerar y operar simultáneamente con la energía y con una propiedad temporal -como lo es la frecuencia- de una y la misma partícula elemental. Y, lo que es más, la ecuación exige inevitablemente que se tomen valores precisos de ambas magnitudes; lo cual, de acuerdo con la relación de incertidumbre, es justamente imposible hacer con precisión respecto a la energía y al tiempo. Así, también aquí nos enfrentamos a una contradicción insostenible: la imposibilidad de determinar precisa y simultáneamente la energía y el tiempo; acompañada de la necesidad, imposible de eludir, de hacer la determinación simultánea y precisa de la energía y de la frecuencia - propiedad temporalde una partícula elemental. Por lo tanto, es conceptualmente posible y experimentalmente necesario determinar a la vez, para una y la misma partícula, la magnitud de su energía y de una de sus propiedades temporales. Esto conduce a plantear la solución de la paradoja en el mismo sentido que en el caso anterior, es decir, considerando a la energía y a la frecuencia u oscilación ondulatoria como aspectos distintos e inseparables de un mismo modo de existencia de las partículas atómicas, que es lo que llamamos sus propiedades temporales. ${ }^{30}$ Entonces, podemos enunciar esta conjugación de la siguiente manera: Respecto a las partículas elementales, el tiempo se puede manifestar como oscilación, o como energía, o bien, como oscilación y energía a la vez. De este modo quedarían satisfechas simultáneamente las relaciones de incertidumbre y la ecuación que conjuga la energía con la frecuencia del movimiento ondulatorio.

30 Como lo ha puesto anteriormente al descubierto la teoría de la relatividad, el tiempo se encuentra ligado a la energía por el mismo sistema que enlaza al espacio con la cantidad de movimiento. 


\section{Espacio y tiempo}

La constitución corpuscular y el movimiento ondulatorio son dos aspectos, contradictorios e indisolubles, de una y la misma realidad: la naturaleza de las partículas elementales. El corpúsculo, al cual se refieren la energía y la cantidad de movimiento, representa una masa puntual de dimensiones cuánticas; esto es, cuya extensión es sumamente pequeña pero finita. En cambio, el movimiento ondulatorio, que es expresado por la frecuencia y la longitud de onda, se extiende de manera continua e infinita en el espacio y en el tiempo. ${ }^{31}$ Difícilmente se podrían concebir dos nociones que, al parecer, fuesen menos susceptibles de enlace que éstas. Y, no obstante, la onda y el corpúsculo se encuentran conjugados realmente en la síntesis unitaria e indivisa de la partícula elemental. Esta conjugación se muestra con claridad en las relaciones existentes entre la energía y la cantidad de movimiento -que caracterizan al aspecto corpuscular-, lo mismo que entre la frecuencia y la longitud de onda - que caracterizan al aspecto ondulatorio- y entre estas cuatro magnitudes a la vez -que caracterizan conjuntamente a la partícula elemental-. Lo cual significa que espacio, tiempo, energía y cantidad de movimiento están enlazados entre sí de todas las maneras posibles - teórica y experimentalmente- tal como se expresa en las ecuaciones fundamentales de la mecánica cuántica. ${ }^{32}$ Más todavía, la mera consideración de la velocidad de una partícula elemental trae consigo la necesidad de considerar la conjugación del espacio con el tiempo. ${ }^{33}$ Por otra parte, como las velocidades a que se mueven las partículas elementales se aproximan a la velocidad de la luz, es indispensable introducir las leyes relativistas en el dominio de los procesos atómicos; $y$, por ende, resulta que

31 Por lo tanto, la descripción ondulatoria de una partícula atómica implica indispensablemente el hacer a la vez determinaciones de lugar y de tiempo, tal como su descripción corpuscular impone la necesidad de hacer determinaciones simultáneas de cantidad de movimiento y de energía.

32 Las seis relaciones posibles se cumplen efectivamente en las ecuaciones de la manera siguiente:

entre la energía y la cantidad de movimiento: $\mathrm{E}=\mathrm{pc}$

entre la frecuencia y la longitud de onda: $v=\frac{c}{\dot{\lambda}}$

entre la cantidad de movimiento y la longitud de onda: $p=\frac{h}{\lambda}$

entre la longitud de onda y la energía: $\lambda=\frac{h c}{E}$

entre la energía y la frecuencia: $E=h v$

entre la cantidad de movimiento y la frecuencia: $p=\frac{h v}{c}$.

33 La velocidad de un objeto cualquiera es el espacio que recorre dicho objeto en la unidad de tiempo. 
sus propiedades no se pueden determinar sólo en el espacio, sino que tienen que ser determinadas simultáneamente con el tiempo. O sea, que es indispensable considerar el espacio-tiempo relativista, al menos como una hipótesis susceptible de modificaciones ulteriores.

Ahora bien, la existencia del cuanto de acción implica una especie de interdependencia entre la localización de una partícula elemental en el espacio y en el tiempo y la especificación de su estado dinámico. Esta interdependencia es expresada por las relaciones de incertidumbre y tiène un carácter nuevo y completamente extraño a las concepciones de la física clásica. Ya la teoría de la relatividad generalizada señala que las propiedades locales del espaciotiempo dependen de la distribución de masa y energía en la región considerada. Pero, lo que el conocimiento de los procesos atómicos plantea es ir todavía más allá de estas condiciones de mutua influencia entre el espaciotiempo y la distribución estática de energía y masa, para incluir la consideración del estado dinámico de las partículas elementales. Esto apunta la necesidad de conjugar el aspecto cinemático y el aspecto dinámico, junto con la oposición entre ellos que está contenida en el cuanto de acción. Las propiedades espaciales de las partículas atómicas no se pueden representar rigurosamente en el espacio-tiempo clásico. $\mathrm{Y}$, al parecer, tampoco la forma espacio-temporal de representación de la teoría de la relatividad sirve estrictamente para explicar dichas propiedades espaciales. Sin embargo, teniendo en cuenta el camino seguido para establecer el espacio-tiempo relativista, parece ser justo el considerar conjuntamente otras propiedades de las partículas; lo cual es indicado, además, de modo confluyente, por los propios resultados de la mecánica cuántica. En todo caso, el espacio y el tiempo de la física clásica - bien definidos y perfectamente utilizables en la escala macroscópica- cesan de ser estrictamente adecuados para la descripción de la realidad física en la escala de los átomos. La teoría cuántica sugiere que estos conceptos aproximados tienen que ser abandonados en el dominio atómico; en forma análoga a como el concepto de temperatura -que es simplemente la manifestación de la agitación molecular- pierde su significado cuando se llega al nivel de las propias moléculas y, más aún, cuando se trata de los átomos o de las partículas que los constituyen.

En el continuo espacio-tiempo relativista, la cantidad de movimiento y la energía están fundidas en una unidad, como lo están el tiempo y el espacio. Por lo tanto, existe un vector tetradimensional en el cual se encuentran conjugados el espacio y el tiempo $(x, y, z, t)$. Igualmente, existe otro vector tetradimensional cuyas magnitudes conjugadas son las componentes de la cantidad de movimiento y la energía con signo negativo, $\left(\mathrm{p}_{\mathbf{x}}, \mathrm{p}_{\mathrm{y}}, \mathrm{p}_{\mathrm{z}},-\mathrm{E}\right)$. Pues bien, conforme a la "complementariedad", es imposible enfocar simultáneamente los dos vectores anteriores. Cuando se determinan con precisión la cantidad de movimiento y la energía de una partícula elemental 
-esto es, las características de su aspecto corpuscular-, entonces la partícula se hace difusa a través de una enorme región del espacio, perdiéndose casi por completo su aspecto corpuscular. Asimismo, cuando se determina con precisión la posición de la partícula elemental en un instante dado -es decir, las características de su aspecto ondulatorio-, entonces se tiene una imprecisión extrema de la cantidad de movimiento y la energía de la propia partícula, con la consiguiente manifestación de la existencia de su aspecto corpuscular. Así, tenemos que la localización espacio-temporal de la partícula elemental $\longrightarrow$ sea, de sus condiciones cinemáticas- y la indicación de su estado de movimiento -es decir, de sus condiciones dinámicas- parecen ser dos planos diferentes de la realidad que no es posible enfocar con precisión al mismo tiempo. Lo que es más, cuando se enfoca uno de estos planos con la mayor exactitud, se observa el otro plano con nitidez; y, a la vez, cuando el enfoque se hace sobre el otro plano, entonces se observa claramente el primero. Por lo tanto, no se trata realmente de una exclusión mutua sino, al contrario, de una conexión estrecha e indisoluble. Como ya lo hemos señalado, el tiempo y la energía son aspectos de una y la misma forma de existencia y, a su vez, el espacio y la cantidad de movimiento son dos aspectos de la misma propiedad fundamental de las partículas elementales. Este enlace es tan íntimo, que la conservación de la energía se puede interpretar indistintamente como conservación de la frecuencia de su movimiento ondulatorio; y, también, la conservación de la cantidad de movimiento se puede interpretar como conservación de la longitud de onda del mismo movimiento. De tal manera que, en resumidas cuentas, debemos considerar la síntesis de las dos parejas de magnitudes, para formar una sola unidad continua de espacio-cantidad de movimiento-energía-tiempo. Esta síntesis estará caracterizada por el cumplimiento riguroso del principio de la conservación de la energía y de la cantidad de movimiento. Entonces, en la conjugación dialéctica de las propiedades fundamentales de las partículas atómicas, tendremos que éstas se manifestarán indistintamente por la conservación de su extensión, de su cantidad de movimiento, de su energía y de su oscilación y, al mismo tiempo, por la mutua transformación entre estas cuatro formas de su existencia.

\section{Dimensionalidad}

De acuerdo con la formulación ondulatoria de la mecánica cuántica, las ondas de una sola partícula elemental se propagan en un espacio de tres dimensiones. En cambio, cuando se trata de dos partículas que se interactúan, sus ondas se propagan en un espacio de seis dimensiones; $y$, en el caso de un millón de partículas en interacción, la propagación de sus ondas ocurre en un espacio de tres millones de dimensiones. En general, para 
representar matemáticamente la propagación ondulatoria de $n$ partículas en

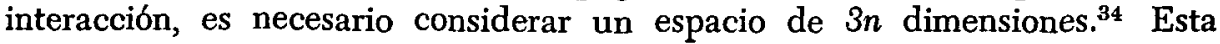
extraña necesidad de la mecánica ondulatoria lleva inmediatamente a suscitar la objeción de la incongruencia que resulta el sostener, por una parte, la realidad física de las ondas - conforme a la representación matemática que ofrece de ellas la propia mecánica ondulatoria- $y$, por otro lado, tener que utilizar un tipo de "espacio matemático" que es enteramente ficticio, puesto que no constituye representación física alguna de la realidad objetiva. No obstante, esto no impide el tener a la forma ondulatoria de la física cuántica como una primera aproximación teórica hacia la explicación de los procesos atómicos que, por lo demás, sirve como fructuosa hipótesis de trabajo para las investigaciones. En realidad, lo que ocurre es que las ondas de las partículas elementales son simplemente -dentro del aparato matemático de la mecánica ondulatoria - representaciones diagramáticas de probabilidades. Esto explica por qué es necesario trabajar con un hiperespacio que tenga el triple de dimensiones del número de partículas atómicas existentes en el sistema considerado. Ahora bien, debemos tener en cuenta que los "espacios matemáticos" de más de tres dimensiones constituyen únicamente un instrumento matemático definido para estudiar conjuntos de objetos, con respecto a un número cualquiera de sus propiedades comunes; las cuales son representadas justamente, de manera simbólica, por el número correspondiente de "dimensiones". Pero este tipo de espacios polidimensionales no tiene una significación física espacialmente objetiva ${ }^{35}$ y, por lo tanto, la necesidad de su empleo señala sencillamente que es indispensable considerar conjuntamente con las cualidades espaciales de las partículas elementales, esas otras propiedades temporales y dinámicas que están conectadas inseparablemente con ellas. En consecuencia, nos encontramos aquí con otro indicio poderoso de la urgente necesidad de establecer una especie de continuo espacio-cantidad de movimiento-energía-tiempo, dentro del cual sean co-eficientes estas cuatro propiedades.

\section{Caracteres cuánticos}

Con el propósito de encontrar una explicación satisfactoria de los procesos de emisión y absorción de energía radiante en los átomos, Bohr propuso la introducción de una condición limitante respecto al movimiento de los electrones atómicos. Con arreglo a esta condición, los electrones solamen-

34 Por lo contrario, cuando se trata de fotones - partículas que no se actúan mutuamente- su propagación ondulatoria se puede representar siempre en un espacio de tres dimensiones, cualquiera que sea el número de fotones considerados.

35 Puesto que la transformación de un espacio polidimensional cualquiera en el espacio tridimensional, aunque es posible, no resulta ser completamente unívoca y continua y, además, hace que se pierda la continuidad de la conexión causal. 
te se pueden mover alrededor del núcleo describiendo ciertas trayectorias privilegiadas - llamadas "órbitas cuánticas"- cuyas distancias al núcleo atómico forman una serie de valores discretos. Cada uno de los términos de esta serie cumple con la exigencia de que la cantidad de movimiento angular resultante para el electrón sea un múltiplo entero de la constante $\frac{\mathrm{h}}{4 \pi}$ o sea, la misma magnitud finita que expresa el límite de la precisión en las relaciones de incertidumbre ${ }^{36}$ Esta condición crítica, sugerida primero como una hipótesis provisional sin fundamento teórico, se ha convertido después - tanto en la mecánica ondulatoria, como en la mecánica de las matrices y en la teoría relativista atómica - en una consecuencia directa de las ecuaciones que representan el conocimiento logrado sobre los procesos atómicos. Por lo tanto, ahora se encuentra explicada consecuentemente la existencia de estas órbitas cuánticas privilegiadas. Es más, de una manera coincidente, la consideración ondulatoria del movimiento de los electrones lleva a descubrir que sus trayectorias atómicas tienen necesariamente longitudes que son múltiplos enteros de la longitud de onda de los propios electrones. O sea, que el movimiento orbital y el movimiento ondulatorio no son independientes. ${ }^{37}$ De esta manera, las órbitas discretas de los electrones son explicadas por una condición de estabilidad impuesta por la continuidad de su movimiento ondulatorio; $y$, por consiguiente, esta explicación constituye una superación dialéctica de la oposición que se plantea entre la continuidad ondulatoria y la discontinuidad cuántica, en uno de sus aspectos.

Ahora bien, a cada una de estas órbitas cuánticas le corresponde una energía de magnitud definida. Por ende, esta energía únicamente puede asumir valores discretos - llamados "niveles de energía" - cada uno de los cuales corresponde a un estado diferente del átomo - "estado cuántico"- caracterizado por el movimiento del electrón en una de las órbitas cuánticas posibles. En estas condiciones, tenemos que la localización espacial de las órbitas electrónicas está especificada por la cuantización de la cantidad de movimiento, por la cuantización de la energía y por la dimensión finita de la longitud de onda de los electrones mismos. ${ }^{38}$ A la vez, esta especificación espacial de las órbitas electrónicas tiene un carácter enteramente cuántico, ya que existe una estricta discontinuidad entre las distancias de las órbitas al

36 En el caso más simple, del átomo de hidrógeno, la distancia del núcleo a la b́rbita electrónica más cercana es de: $0.527 \times 10^{-8} \mathrm{~cm}$.

37 Como se advierte, sucede lo contrario de lo que ocurre entre el movimiento de translación y el movimiento de rotación de un planeta, ya que el recorrido de una vuelta completa alrededor del sol no coincide con un número entero de rotaciones del mismo planeta.

38 Téngase presente que, para una velocidad constante, a la dimensión finita de la longitud de onda le corresponde una dimensión también finita de la oscilación temporal o frecuencia. 
núcleo atómico -que se encuentran dosificadas como múltiplos enteros de ja distancia mínima - y, por lo tanto, asimismo existe una rigurosa discontinuidad en la longitud de las propias trayectorias orbitales. Por otra parte, tenemos la consideración de que el paso del electrón de una órbita a otra se produce bruscamente, como un "salto cuántico". Lo único que se explicita para este cambio repentino de una órbita a otra es que en su realización se cumple con el principio de la conservación de la energía y de la cantidad de movimiento. En consecuencia, el átomo absorbe o emite la energía correspondiente a la diferencia entre los dos niveles en que se produce. ${ }^{39}$ Esta energía es absorbida o emitida ordinariamente en forma de radiación y su mag. nitud está conectada con su frecuencia. A su vez, las frecuencias de la radiación se manifiestan como "términos espectrales"; correspondiendo cada término a un nivel de energía diferente $y$, por lo tanto, a un estado cuántico distinto. ${ }^{40}$

Por lo anterior, tenemos que el espacio interno de los átomos es de naturaleza cuántica y, por ende, discontinua. De un modo aproximado, lo podemos representar como una sucesión de lugares geométricos continuós - las órbitas cuánticas posibles- separados por extensiones discontinuas en donde jamás se pueden encontrar los electrones. Y, sin embargo, estas extensiones discontinuas que separan a las órbitas estables permiten el paso de los electrones de una órbita a otra, en forma de saltos bruscos. Es decir, que el enlace dinámico entre las trayectorias continuas se efectúa a través de la discontinuidad que media entre ellas. Así, resulta que el espacio interno del átomo está constituído por trozos de continuidad -las trayectorias electrónicas- cuyo enlace se establece, de manera interminente y súbita, por intermedio de trozos discretos en donde es imposible la permanencia de los electrones. Estos saltos bruscos de una órbita a otra establecen justamente una solución de continuidad, que impide su representación en un espacio continuo y que tampoco corresponde a una continuidad del tiempo. Por lo demás, en los trozos continuos en donde sí es posible la permanencia de los electrones, la posición de un electrón - puesto que su órbita es un lugar geométrico- se encuentra enteramente determinada por su cantidad de movimiento; y, a la inversa, la cantidad de movimiento de un electrón está determinada completamente por su posición orbital - que incluye su distancia discreta al núcleo y la dimensión definida de la misma trayectoria-. En consecuencia, a cada cantidad de movimiento angular - que sea un múltiplo entero de la constante cuántica $\frac{h}{4 \pi}-$ le corresponde una órbita electrónica

39 Hay absorción de energía cuando la transición es de una órbita interna a un nivel externo de mayor energía; y hay emisión en el caso opuesto.

40 Esta teoría explica completamente más de 50 de los términos observados en el espectro del átomo de hidrógeno y en los espectros de los átomos ionizados semejantes al hidrógeno. 
cuya posición está bien definida; y, recíprocamente, a la posición cuántica de cada órbita electrónica posible le corresponde una cantidad de movimiento angular precisamente determinada. Nuevamente nos encontramos aquí con la conjugación dialéctica objetiva de la posición y la cantidad de movimiento de las partículas atómicas.

\section{Discontinuidad cinemática}

Si en un punto $A$ se encuentra una fuente luminosa en donde ocurre un proceso de emisión elemental y en otro punto $B$ hay una partícula que termina un proceso de absorción elemental, entonces parece posible considerar que el fotón emitido en $A$ ha viajado en línea recta desde este punto hasta $B$, en donde ha sido absorbido. Pero esta consideración sobre la rectilinearidad de la trayectoria se modifica cuando colocamos una pantalla opaca con un orificio pequeño entre los puntos $A$ y $B$. Ya que, entonces, debido al fenómeno de difracción, puede ocurrir que el fotón no llegue al punto $B$, aunque el orificio esté alineado con $A$ y con $B$, o bien, puede suceder que el fotón llegue a $B$, a pesar de que el orificio no se encuentre sobre la recta que va de $A$ a $B$. Si repetimos este procedimiento, interponiendo otras muchas pantallas opacas con hendeduras pequeñas en distintos puntos entre $A$ y $B$, podremos advertir cómo la trayectoria se seguirá quebrando cuanto queramos, de tal manera que será imposible tenerla localizada con precisión. Entonces, tenemos que únicamente se puede determinar con precisión la salida de un fotón y su llegada, o sea su emisión y su absorción; pero, en cambio, no se puede determinar rigurosamente la trayectoria que sigue entre su emisión y su absorción. La emisión de un fotón es un proceso que presenta analogía con el disparo de un proyectil: hay retroceso y pérdida de energía. A su vez, la absorción de un fotón tiene analogía con el impacto de un proyectil. Es más, en el caso simple en que no existe otro obstáculo entre el punto de emisión y el de absorción, el fotón es absorbido justamente después de que ha transcurrido el tiempo necesario para recorrer la distancia que separa dichns puntos a la velocidad de la luz. Sólo que, a pesar de estas analogías, no existe continuidad en la trayectoria seguida por el fotón. Esto significa que la continuidad de la trayectoria tiene independencia lógica respecto a las otras propiedades señaladas. $Y$, al mismo tiempo, nos permite advertir que esta independencia logica se ha descubierto como resultado del conocimiento experimental y no, en modo alguno, como producto exclusivo de la reflexión racional.

Como la partícula elemental se manifiesta únicamente cuando reacciona con otras partículas, entonces se muestra sólo de trecho en trecho $y$, por consiguiente, no se le puede atribuir una trayectoria continua. Así, la mecánica cuántica se limita a vincular entre sí las diversas manifestaciones sucesivas y discontinuas de la existencia espacial de una partícula, sin poder pre- 
cisar lo que ocurre en el intervalo. O sea, que las propiedades espaciales de la partícula elemental se exhiben solamente de un modo intermitente. Tampoco es posible establecer una determinación espacial continua de las partículas elementales dentro de los átomos. Porque, para definir físicamente la trayectoria del electrón, sería necesario poder determinar su posición en instantes sucesivos - con incertidumbres pequeñas comparadas con las dimensiones de su órbita - y repetir la observación sobre el mismo electrón muchas veces. Pero, para ejecutar una observación de posición con una incertidumbre pequeña comparada con las dimensiones de la órbita es ineludible, debido al principio de incertidumbre, impartir al electrón un cambio er. su cantidad de movimiento. Y, entonces, en la siguiente observación su movimiento se encuentra ya perturbado. Por lo tanto, lo único posible es hacer una determinación aproximada de la órbita, procurando que la perturbación producida sea despreciable comparada con la cantidad de movimiento que el electrón posee en su movimiento orbital. Los complementaristas interpretan esta situación en el sentido de que el concepto de "órbita" y el de "estado cuántico" son magnitudes complementarias y, por ende, excluyentes. En rigor, como ya lo hemos hecho ver, se trata nuevamente de magnitudes incluyentes. Con todo, parece obligado el abandono de la consideración de trayectorias continuas para el movimiento de las partículas elementales. Ya que, aun cuando en algunos casos se puedan especificar experimentalmente dos puntos de la trayectoria de una partícula elemental, sin embargo, no es consecuente - por los hechos antes apuntados- considerar con rigor que el intervalo recorrido entre esos dos puntos determinados sea continuo. Y, por lo tanto, tenemos que aceptar la posibilidad de que exista una discontinuidad cinemática para el espacio en el nivel atómico de la existencia.

\section{Politopía y homotopía}

De acuerdo con la expresión matemática del movimiento ondulatorio de una partícula elemental, tenemos que el cuadrado de la amplitud de onda -su intensidad - mide en cada punto espacial y en cada instante temporal, la probabilidad de que el corpúsculo se encuentre en ese punto en dicho instante. De este modo, parece posible considerar que la partícula elemental es un fenómeno periódico extendido en el espacio - la onda- y, a la vez, centrado en un punto -el corpúsculo-. Ahora bien, por virtud del principio de exclusión de Pauli, tenemos que dos de las partículas que forman un sistema jamás pueden tener la misma energía, o la misma cantidad de movimiento. ${ }^{41} \mathrm{O}$ sea que, en este sentido, cada partícula llena todo el sistema

41 Esta teoría, incluída en la "estadística de Fermi", es aplicable a los gases formados por electrones y también ha permitido interpretar las propiedades electrónicas y térmicas de los metales. 
por sí sola y, por ende, tiene la propiedad de ser politópica, es decir, que tiene simultáneamente una multitud de localizaciones espaciales diferentes. ${ }^{42} \mathrm{Y}$, al mismo tiempo, como esto ocurre para todas y cada una de las partículas, resulta que éstas tienen también la propiedad de ser homotópicas respecto a cada lugar, puesto que lo ocupan simultáneamente todas ellas. ${ }^{43}$ En el seno del conjunto, las partículas están extendidas de manera continua y reaccionan constantemente entre sí. Dicho de otro modo, una partícula situada en un punto cualquiera del sistema impide que cualquiera otra partícula del propio sistema tenga el mismo estado cuántico que ella. Esta probabilidad de que la presencia de la partícula se manifieste simultáneamente en muchos puntos del espacio - lo cual se debe tanto al principio de exclusión como a la amplitud de la ondatermina con la noción de espacio vacío en el dominio atómico. Esto se antoja completamente paradójico, puesto que el sistema puede suponerse tan grande como se quiera y, en consecuencia, las partículas pueden estar tan alejadas como se quiera. Pero, si se recuerda la localización múltiple de las partículas elementales - es decir, su politopía-, se desvanece el sentido de la lejanía o de la cercanía. ${ }^{44} \mathrm{Al}$ parecer, no existen partículas elementales aisladas, abandonadas a sí mismas, "libres". Porque, incluso cuando se encuentran a una distancia considerable, las partículas siguen conectadas con el medio al cual pertenecen. Por este enlace indisoluble entre la partícula elemental y su medio, se pone al descubierto la imposibilidad de aislarla, tal como queda señalado con claridad dentro de los tres distintos aparatos teóricos de la mecánica cuántica.

Por otra parte, tenemos la consideración de que la fuerza que vincula a los átomos de una molécula de hidrógeno $-\mathrm{H}_{2}$, diatómica- se debe al intercambio de los estados cuánticos de los dos electrones -uno de cada átomo- y, por ello, es denominada "energía de intercambio". Esta energía desempeña una función importante en las interacciones de los átomos próximos que constituyen una molécula o la celosía de un cristal y, posiblemente, también en las acciones recíprocas entre las partículas que integran el núcleo atómico. ${ }^{45}$ La "energía de intercambio" sólo surge cuando dos partículas de tipo idéntico tienen una probabilidad que no es nula de encontrarse en el mismo lugar del espacio en el mismo instante. Es decir, que únicamente

42 En la física clásica todas las partículas son monotópicas, ya que solamente pueden tener una localización espacial en cada instante.

43 Las partículas de la mecánica clásica son siempre heterotópicas, puesto que un mismo lugar nunca puede ser ocupado por varias partículas al mismo tiempo.

44 Esta variación de la distancia ya se muestra claramente en cualquier geometría que no sea métrica.

45 Cuando se estudia la evolución dinámica de un sistema que contiene partículas del mismo tipo, con arreglo a la mecánica cuántica, se advierte que, al lado de los términos que expresan la existencia de las interacciones conocidas entre las partículas, aparecen otros términos que se interpretan como representativos de esta "energía de intercambio" que constituye el vínculo entre las partículas homopolares. 
existe "energía de intercambio" cuando dos partículas elementales de la misma especie tienen dominios de presencia posible que encajan el uno en el otro, o sea, cuando se manifiesta la propiedad de homotopía entre ellas. Por lo demás, esta posibilidad de que varias partículas ocupen el mismo lugar al mismo tiempo, está ligada también con el problema de la carencia de individualidad que exhiben las partículas elementales $y$, por consiguiente, con la cuestión de la identificación completa entre las partículas del mismo tipo.

Ahora bien, en el enfoque de la mecánica ondulatoria, la partícula elemental está representada por una onda compleja definida por cierta descomposición espectral y cuya amplitud está repartida en cada instante de manera determinada en el espacio. Como ya lo expresamos, el corpúsculo puede manifestar su presencia en un punto cualquiera de la región ocupada por la onda, con una probabilidad que es proporcional al cuadrado de la amplitud de la onda en ese punto. A la vez, a cada componente monocromático que figura en la descomposición espectral de la onda le corresponde un valor posible de la cantidad de movimiento del corpúsculo. En el caso de una onda simple - monocromática- le corresponde un corpúsculo cuya cantidad de movimiento es perfectamente conocida. Pero, entonces, la onda tiene una cxtensión indefinida en el espacio y en todos los puntos posee la misma amplitud. Por lo tanto, el corpúsculo tiene una posición indeterminada y puede encontrarse en cualquier punto de la región espacial ocupada por la onda y, por consiguiente, se muestra con una politopía homogénea. Ahora bien, cuando la onda ocupa una región sumamente pequeña del espacio, fuera de la cual la amplitud sea nula - es decir, cuando se trata de una onda puntual-, - entonces la posición del corpúsculo es bien conocida. Pero, en tal caso, dicha onda es el resultado de la superposición de ondas monocromáticas de todas las longitudes de onda posibles. $\mathrm{Y}$, por lo tanto, son posibles todos los valores para la cantidad de movimiento de la partícula. O sea, que cuando hay certidumbre acerca de la posición, existe completa incertidumbre sobre la cantidad de movimiento, y viceversa. En otras palabras, la precisión de la localización de una partícula es un resultado de la superposición de ondas y, en consecuencia, trae aparejada la homotopía de muchas partículas. Y, a su vez, la precisión de la cantidad de movimiento es un resultado de la politopía homogénea de una partícula, que se produce en el caso de una onda simple.

\section{Geometría discontinua}

Por las consideraciones relatadas, podemos afirmar que el conocimiento científico adquirido sobre los procesos físicos atómicos ha puesto en crisis tanto la concepción clásica del espacio como su concepción relativista. En buena parte, la insuficiencia mostrada por las interpretaciones clásica y rela- 
tivista del espacio se debe al carácter rígidamente continuo del marco que ambas ofrecen para la representación espacial de los procesos atómicos. De aquí que se suscite la cuestión de explorar las posibilidades de que el marco discontinuo que se requiere para la representación de las propiedades espaciales de las partículas atómicas pueda ser expresado por alguno de los tipos de geometría que ya se encuentran desarrollados matemáticamente. Pues bien, parece ser que no resulta aplicable la geometría métrica, ni tampoco la homotésica, la afín o la proyectiva. Y, lo que es más, ni siquiera la geometría más general, la topología - que se ocupa justamente de las transformaciones que son reversibles, biunívocas y continuas- parece ofrecer la estructura espacial necesaria para la representación de los procesos atómicos. Entonces, tomando en cuenta que las propiedades topológicas son invariantes a toda suerte de distorsiones con excepción de la ruptura, ${ }^{46}$ cabe preguntar si será indispensable la construcción de una geometría todavía más general que la topología, en la cual se pueda "romper" el espacio sin perjuicio de que se mantengan invariantes algunas de sus propiedades. Esto es, con mayor precisión, ¿será menester el establecimiento de una geometría que estudie las transformaciones discontinuas? La respuesta no la podemos dar ahora. En todo caso, requiere previamente una elaboración matemática bien difícil. Por lo cual nos tenemos que contentar aquí con sugerir el planteamiento del problema.

\section{Espacio atómico}

Con base en todo lo anteriormente expuesto, podemos presentar las modalidades que asume la categoría de espacio en la física atómica. Desde luego, tenemos que la relación espacial es una de las formas primordiales en que se manifiesta la concatenación existente entre los procesos atómicos. Y esta concatenación permite advertir, a su vez, que el espacio no es algo independiente de los procesos, ni tampoco constituye una especie de recipiente en el cual estuviesen inmersas las partículas elementales. Simplemente, el espacio es el conjunto de las propiedades espaciales que son inherentes a los procesos atómicos y representan una forma de su existencia. Por lo tanto, los procesos atómicos no existen en el espacio, sino que su existencia es espacial. Más aún, el carácter común que tienen las propiedades espaciales exhibe de manera definida la pertenencia inextinguible de cada partícula elemental a su medio $y$, por consiguiente, la conexión entre todas las partículas. $\mathrm{Y}$, por lo demás, esta conexión espacial se manifiesta de modo rigurosamente tridimensional.

46 Felix Klein, Elementary mathematics from an advanced standpoint: geometry, Macmillan, New York, 1939; págs. 105-109. 
Por otra parte, tenemos que el espacio atómico posee dos aspectos diferentes pero distintos, que son la extensión y la cantidad de movimiento. Análogamente, el tiempo atómico tiene dos formas diferentes y no distintas, que son la oscilación y la energía. Además, el espacio y el tiempo constituyen un todo único inseparable. De tal manera que, tomándolos conjuntamente, se forma la unidad espacio-cantidad de movimiento-energía-tiempo. En consecuencia, se puede establecer el siguiente juicio complejo: Respecto a las partículas elementales, la existencia objetiva se puede manifestar: 1) como extensión; 2) como cantidad de movimiento; 3) como energía; 4) como oscilación; 5) como extensión y cantidad de movimiento; 6) como extensión y energía; 7) como extensión y oscilación; 8) como cantidad de movimiento v energía; 9) como cantidad de movimiento y oscilación; 10) como energía y oscilación; 11) como extensión, cantidad de movimiento y energía a la vez; 12) como extensión, cantidad de movimiento y oscilación a la vez; 13) como extensión, energía y oscilación a la vez; 14) como cantidad de movimiento, energía y oscilación a la vez; y, 15) simultáneamente como extensión, cantidad de movimiento, energía y oscilación. En la conjugación de estas propiedades fundamentales de las partículas atómicas se cumple estrictamente el principio de conservación - para cada una de ellas y para todos sus enlaces- $y$, a la vez, se cumple igualmente el principio de transformación de unas en otras. De manera destacada, aunque no exclusiva, el espacio-cantidad de movimiento representa la conservación de las cualidades en las partículas atómicas. Y, también de modo conspicuo pero no privativo, el tiempo-energía es el exponente de la transformación de esas cualidades y del surgimiento de otras nuevas.

Por otro lado, el espacio atómico se muestra como existencia continua, en un conjunto simple e inseparable; pero, al mismo tiempo, pone de manifiesto su discontinuidad y su estructura de naturaleza cuántica. La continuidad se exhilse acentuadamente en el movimiento ondulatorio $y$, por ende, en la extensión y la oscilacion; mientras que la discontinuidad corresponde notablemente al corpúsculo $y$, por consiguiente, a la cantidad de movimiento y a la energía. Sin embargo, existe también una transposición constante de estos aspectos contradictorios, de manera tal que la extensión y la oscilación muestran su carácter discontinuo, en tanto que la energía y la cantidad de movimiento exhiben su continuidad. En el interior del átomo, el espacio manifiesta su naturaleza cuántica en las longitudes: en las distancias del núcleo a las órbitas electrónicas y en las dimensiones de las propias trayectorias. $Y$ esta cuantización espacial se encuentra en mutua dependencia con la cuantización de la cantidad de movimiento, de la energía y del tiempo. A la vez, las trayectorias electrónicas son lugares geométricos continuos, separados por trozos discretos. En estas trayectorias, la coincidencia exacta entre su longitud y su número entero de oscilaciones ondulatorias, constituye una 
conjugación de la discontinuidad y la continuidad del espacio. Y el enlace entre las órbitas cuánticas se establece por el cambio de la energía, absorbida o emitida, que supera así las soluciones de continuidad. En el exterior del átomo, las partículas elementales se muestran en trayectorias corpusculares que son espacialmente discontinuas y temporalmente intermitentes. A la vez, en su movimiento ondulatorio, las partículas atómicas se manifiestan de manera continua y persistente, tanto espacial como temporalmente. A más de esto, la interacción de las partículas elementales constituye el enlace indisoluble de continuidad entre todas ellas. En fin, la conexión universal entre todos los lugares del espacio atómico se muestra del modo más acusado en las peculiares propiedades de homotopía y politopía de las partículas elementales.

Tales son los rasgos característicos de la categoría de espacio en la física atómica, que intentan explicar de modo racional y objetivo las formas espaciales de existencia de las partículas elementales. Independientemente del acierto o del desacierto de la formulación que hemos hecho, es obvio que esta categoría cambia notablemente en este nivel de la existencia. Pero la indudable profundización del conocimiento científico lograda por la física atómica no ha alterado, en manera alguna, la objetividad del espacio como forma fundamental de la existencia del universo. Por el contrario, es esa mayor penetración en el conocimiento del espacio objetivo la que ha producido todas las modificaciones que aquí constatamos en su concepción. Y, casi por demás está decir, que nuestra construcción entera es simplemente una hipótesis sobre la categoría de espacio en el dominio de las partículas elementales, que tendrá que ser sometida a la prueba decisiva de la verificación experimental.

Elr de Gortari 